\title{
Influência da Clonidina por Via Venosa no Custo de Anestesia com Sevoflurano em Cirurgias de Ouvido Médio em Regime Ambulatorial *
}

\section{Influence of Intravenous Clonidine in the Cost of Sevoflurane Anesthesia for Outpatient Middle Ear Procedures}

Renato Mestriner Stocche, TSA ${ }^{1}$; Luís Vicente Garcia, TSA ${ }^{1}$; Jyrson Guilherme Klamt, TSA ${ }^{1}$; Marlene Paulino dos Reis ${ }^{2}$, Daniela Rocha Gil ${ }^{3}$; Karin Luiza Magno Mesquita ${ }^{3}$

\section{RESUMO}

Stocche RM, Garcia LV, Klamt JG, Reis MP, Gil DR, Mesquita KLM - Influência da Clonidina por Via Venosa no Custo de Anestesia com Sevoflurano em Cirurgias de Ouvido Médio em Regime Ambulatorial

JUSTIFICATIVA E OBJETIVOS: A clonidina é um agente $\alpha_{2}-$ agonista que diminui o consumo de anestésicos venosos e inalatórios. Este estudo visou avaliar a relação custo-benefício da medicação pré-anestésica com clonidina por via venosa em anestesia geral com sevoflurano em regime ambulatorial.

MÉTODO: Trata-se de estudo encoberto, aleatório, controlado com placebo, realizado com pacientes com idade entre 15 e 52 anos. Os pacientes foram divididos em 3 grupos de 15: Grupo $S$ (placebo), Grupo C3 (clonidina $3 \mu \mathrm{g} \cdot \mathrm{kg}^{-1}$ ) e Grupo C5 (5 $\left.\mu \mathrm{g} . \mathrm{kg}^{-1}\right)$. A indução anestésica foi feita com sevoflurano, alfentanil $\left(30 \mu \mathrm{g} \cdot \mathrm{kg}^{-1}\right)$ e pancurônio $\left(0,08 \mathrm{mg} \cdot \mathrm{kg}^{-1}\right)$. Foram anotados a freqüência de complicações, consumo de halogenados, tempo de anestesia, tempo de recuperação fase I e II. A análise de custos considerou gastos diretos e indiretos.

RESULTADOS: Não houve diferenças entre os grupos em relação aos dados demográficos, freqüência de complicações e tempo para recuperação anestésica fase I. A recuperação anestésica fase II foi prolongada no grupo C5 $(p<0,05)$. O consumo de sevoflurano por minuto de cirurgia foi $0.54 \pm 0,14$ no grupo $S$ e 0,33 $\pm 0,09$ e 0,34 0,13 nos grupos $C 3$ e C5, respectivamente $(p<0,05)$. Nos grupos que receberam clonidina, o custo foi diminuído em aproximadamente $35 \%$.

CONCLUSÕES: A clonidina $\left(3 \mu \mathrm{g} \cdot \mathrm{kg}^{-1}\right)$ por via venosa diminui o consumo de sevoflurano sem aumentar o tempo de recuperação fase II. A dose de $5 \mu \mathrm{g} \cdot \mathrm{kg}^{-1}$, apesar de diminuir o consumo de sevoflurano, prolonga a recuperação fase II, não se adequando ao regime ambulatorial.

Unitermos: ANESTESIA: Ambulatorial; ANESTÉSICOS, Volátil: sevoflurano; DROGAS, $\alpha_{2}$ - agonista: clonidina

\footnotetext{
* Recebido do (Received from) Hospital das Clínicas da Faculdade de Medicina de Ribeirão Preto (HC-FMRP - USP), Ribeirão Preto, SP

1. Professor da Disciplina de Anestesiologia da FMRP-USP

2. Professora da Disciplina de Anestesiologia da FMRP-USP

3. Ex-ME do CET do HC da FMRP-USP; Assistente do HCRP-USP
}

Apresentado (Submitted) em 07 de fevereiro de 2003

Aceito (Accepted) para publicação em 05 de maio de 2003

Endereço para correspondência (Correspondence to)

Dr. Renato Mestriner Stocche

Rua Adolfo Serra, 237

Alto da Boa Vista

14025-520 Ribeirão Preto, SP

(C) Sociedade Brasileira de Anestesiologia, 2004

\section{SUMMARY}

Stocche RM, Garcia LV, Klamt JG, Reis MP, Dil DR, Mesquita KLM - Influence of Intravenous Clonidine in the Cost of Sevoflurane Anesthesia for Outpatient Middle Ear Procedures

BACKGROUND AND OBJECTIVES: Clonidine is an $\alpha_{2}$-agonist which decreases intravenous and inhalational anesthetics consumption. This study aimed at evaluating the cost-benefit ratio of preanesthetic medication with intravenous clonidine for general anesthesia with sevoflurane in outpatient procedures. METHODS: Forty five patients aged 15 to 52 years were included in this double-blind, randomized and placebo controlled study, and were distributed in 3 groups of 15: Group $S$ (placebo), Group C3 (3 $\mu \mathrm{g} \cdot \mathrm{kg}^{-1}$ clonidine) and Group C5 (5 $\mu \mathrm{g} \cdot \mathrm{kg}^{-1}$ clonidine). Anesthesia was induced with sevoflurane, alfentanil $\left(30 \mu \mathrm{g} \cdot \mathrm{kg}^{-1}\right)$ and pancuronium $\left(0.08 \mathrm{mg} \cdot \mathrm{kg}^{-1}\right)$. The following parameters were recorded: incidence of complications, halogenate consumption and anesthesia duration, as well as phase I and II recovery time. Cost analysis has considered direct and indirect costs.

RESULTS: There were no differences among groups in demographics data, incidence of complications and phase I anesthetic recovery. Phase II anesthetic recovery was prolonged in Group C5 $(p<0.05)$. Sevoflurane consumption per minute of surgery was $0.54 \pm 0.14,0.33 \pm 0.09$ and $0.34 \pm 0.13$ in Groups $S, C 3$ and $C 5$ respectively $(p<0.05)$. Costs were approximately $35 \%$ lower in the clonidine groups.

CONCLUSIONS: Intravenous clonidine $\left(3 \mu \mathrm{g} \cdot \mathrm{kg}^{-1}\right)$ decreases sevoflurane consumption without prolonging phase I recovery. Although decreasing sevoflurane consumption, $5 \mu \mathrm{g} . \mathrm{kg}^{-1}$ clonidine prolongs phase II recovery, thus being inadequate for outpatient procedures.

Key Words: ANESTHESIA: Ambulatory; ANESTHETICS, Volatile: sevoflurane; DRUGS, $\alpha_{2}$ - agonist: clonidine

\section{INTRODUÇÃO}

$\mathrm{D}$ esde de meados da década de 1980, existe uma tendência mundial de mudança do regime cirúrgico de internação para ambulatorial. A alta hospitalar precoce para pacientes cirúrgicos proporciona maior conforto, autonomia e satisfação para o paciente, além de diminuir o risco de infecção hospitalar e o custo total da internação ${ }^{1}$. Objetivando esta alta precoce, drogas anestésicas de rápida eliminação são utilizadas nesses pacientes. Entretanto, o alto custo dessas drogas podem limitar a sua utilização ${ }^{2}$. A clonidina é uma droga $\alpha_{2}$-agonista de ação central que diminui o tônus do sistema simpático e apresenta ação sedativa, antisialogoga, antiemética e antálgica. A sua segurança na prática anestésica já foi amplamente estudada e confir- 
mada. Quando usada como medicação pré-anestésica, proporciona redução na demanda de anestésicos inalatórios e venosos $^{3,4}$. Em cirurgia ambulatorial, a clonidina por via venosa, na dose de $1,5 \mu \mathrm{g} . \mathrm{kg}^{-1}$, não mostrou efeito ansiolítico ${ }^{5}$. Entretanto, o efeito da medicação pré-anestésica com clonidina nos custos anestésicos em regime ambulatorial não foi avaliado. Portanto, uma análise da relação custo-benefício faz-se necessária ${ }^{6}$.

Este estudo visou avaliar a relação custo-benefício da medicação pré-anestésica com clonidina por via venosa em anestesia geral com sevoflurano em regime ambulatorial.

\section{MÉTODO}

O estudo foi aprovado pela Comissão de Ética, aleatório, controlado com placebo, duplamente encoberto, realizado no Hospital das Clínicas de Ribeirão Preto em pacientes submetidos a cirurgias de ouvido médio que deram consentimento após a devida informação. Os pacientes apresentaram idades entre 15 e 52 anos, estado físico ASA I e II e peso entre 40 e $85 \mathrm{~kg}$. Foram excluídos do protocolo os pacientes com história de alergia às drogas anestésicas utilizadas, pacientes com freqüência cardíaca inicial menor que 50 bpm, bem como pacientes que faziam uso crônico de agentes depressores do SNC, antidepressivos e anticonvulsivantes, dependentes químicos ou de álcool.

Todos os pacientes foram monitorizados com cardioscópio, oxímetro de pulso, capnometria e pressão arterial não invasiva. Após a realização de punção venosa com cateter $20 \mathrm{G}$, a hidratação foi padronizada com $250 \mathrm{ml}$ em infusão rápida seguida de $6 \mathrm{ml} . \mathrm{kg} . \mathrm{h}^{-1}$ de solução de Ringer com lactato.

Os pacientes foram distribuídos aleatoriamente em 3 grupos: Grupo C3, pacientes que receberam clonidina (3 $\left.\mu \mathrm{g} . \mathrm{kg}^{-1}\right)$; Grupo C5, que receberam clonidina $\left(5 \mu \mathrm{g} . \mathrm{kg}^{-1}\right)$; e Grupo S, que não receberam solução de cloreto de sódio a $0,9 \%$ como medicação pré-anestésica. Após o sorteio do grupo a que pertenceria o caso, um anestesiologista não envolvido com a pesquisa preparou as soluções com medicação pré-anestésica contendo clonidina $30 \mu \mathrm{g} \cdot \mathrm{ml}^{-1}$ para o grupo C3, $50 \mu \mathrm{g} \cdot \mathrm{ml}^{-1}$ para o grupo C5 e solução de cloreto de sódio a $0,9 \%$ para o grupo $S$. Para qualquer grupo, estas soluções foram administradas, por via venosa, no volume de $1 \mathrm{ml}$ de solução para cada $10 \mathrm{~kg}$ do paciente.

Após 15 minutos da administração da medicação pré-anestésica, realizou-se indução anestésica inalatória com sevoflurano, inicialmente à concentração de $3 \%$, seguido de aumento de $0,5 \%$ a cada 15 segundos, até a perda do reflexo palpebral. A seguir, foi administrado alfentanil $\left(30 \mu \mathrm{g} \cdot \mathrm{kg}^{-1}\right) \mathrm{e}$ pancurônio $\left(0,08 \mathrm{mg} \cdot \mathrm{kg}^{-1}\right)$. A manutenção anestésica foi rea-

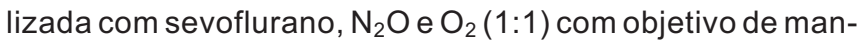
ter a PAM e a FC $20 \%$ a mais ou a menos que a inicial. Todos os pacientes receberam $100 \mathrm{mg}$ de cetoprofeno no início do procedimento.

Ao término da cirurgia e início do curativo, foi administrado oxigênio a $100 \%$ e mudado o sistema respiratório para sem reinalação. A extubação traqueal foi realizada quando o paciente apresentou ventilação espontânea e rítmica, durante pelo menos 30 segundos, mantendo a $\mathrm{P}_{\mathrm{ET}} \mathrm{CO}_{2}$ abaixo de 50 $\mathrm{mmHg}$, além de responder a estímulos verbais. Foi verificado o tempo decorrido entre a interrupção do sevoflurano (término da cirurgia) e a abertura ocular ao comando (solicitado a cada 20 segundos), bem como o tempo entre a interrupção do sevoflurano e a extubação traqueal. Anotou-se também o consumo total de sevoflurano.

As freqüências de eventos adversos foram anotadas. Considerou-se taquicardia a freqüência cardíaca (FC) maior que 100 bpm, bradicardia a FC menor que 50 bpm e hipotensão arterial a pressão menor que $30 \%$ da medida inicial em duas medidas consecutivas. Na SRPA, anotaram-se, também, os eventos adversos (náuseas, vômitos, dor moderada ou forte, retenção urinária, hipotensão arterial postural, sonolência e tontura). Foi utilizada a nebulização com oxigênio a $8 \mathrm{~L}$. $\mathrm{min}^{-1}$ com máscara de Venturi até o paciente apresentar períodos de despertar espontâneo. Pacientes que apresentaram náuseas ou vômitos receberam metoclopramida, somando-se os custos da medicação ao custo final. Verificou-se o tempo levado para condições de alta anestésica ambulatorial pelos critérios descritos por $\mathrm{Chung}^{8}$, a saber, soma de nove pontos seguindo a escala de parâmetros descritos na tabela I.

Tabela I - Critérios Modificados para Alta Anestésica Ambulatorial

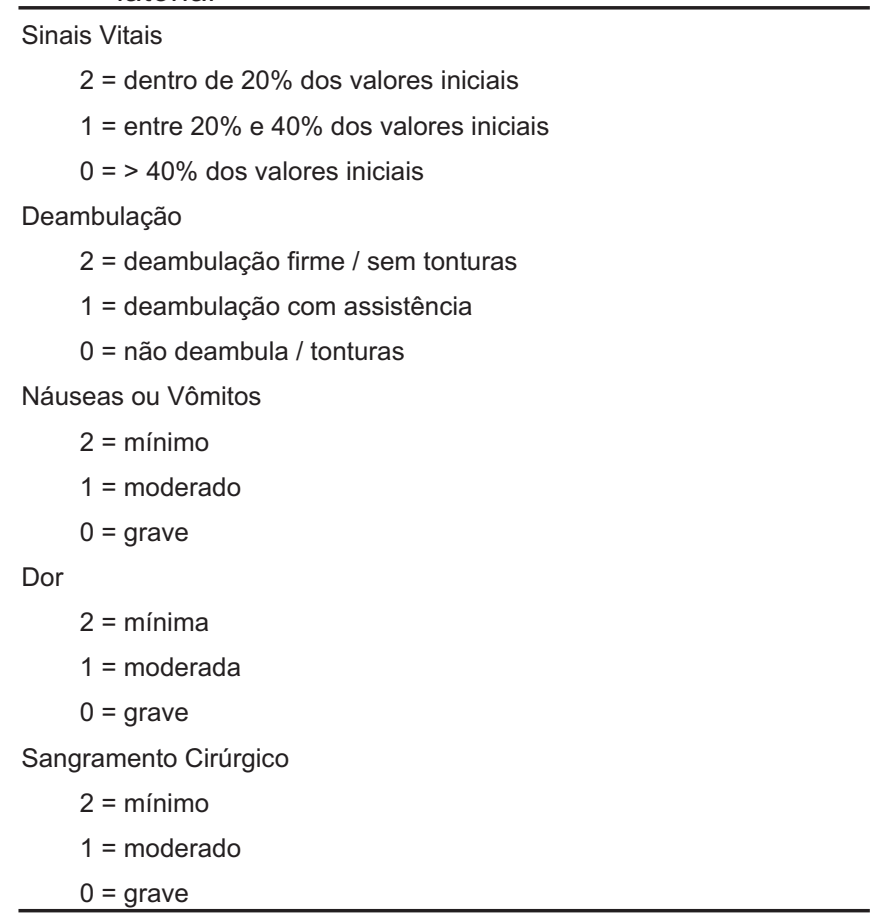

O custo anestésico total foi calculado somando-se todos os gastos com medicações anestésicas, oxigênio, $\mathrm{N}_{2} \mathrm{O}$, materiais descartáveis e custo da permanência na SRPA (minutos). Não foram considerados os custos com depreciação de monitore e ventiladores, bem como os gastos com materiais reutilizáveis (toalhas, coxins, intermediários e outros). O conjunto de materiais descartáveis utilizados continha um tubo traqueal, um cateter venoso $20 \mathrm{G}$, um equipo para soro- 
terapia, um quarto de rolo de esparadrapo, uma sonda plástica para aspiração e dois pares de luvas de látex de procedimento. Os custos com medicações e materiais descartáveis foram obtidos dos valores contidos no Brasíndice, publicado em 20 de julho de 2002, descontando o valor do ICMS embutido no preço. Quando a medicação ou material tinha mais de um fabricante no Brasíndice, o valor considerado nos cálculos foi obtido pela média aritmética dos valores. O custo da hora de internação na SRPA foi obtido no centro de controle de custos do hospital e foi reajustado para o mês de julho de 2002. Já, os valores do metro-cúbico de oxigênio e $\mathrm{N}_{2} \mathrm{O}$ foram obtidos diretamente com o fabricante (valor de tabela) (Tabela II).

Tabela II - Custos de Materiais e Drogas Anestésicas

\begin{tabular}{lc}
\hline Drogas & Preço Médio \\
\hline Alfentanil (ampola de $2,5 \mathrm{mg}$ ) & $\mathrm{R} \$ 15,29$ \\
Sevoflurano (ml) & $\mathrm{R} \$ 2,62$ \\
Pancurônio (ampola de $4 \mathrm{mg}$ ) & $\mathrm{R} \$ 4,88$ \\
Atropina (ampola $0,25 \mathrm{mg}$ ) & $\mathrm{R} \$ 0,45$ \\
Prostigmina (ampola $0,5 \mathrm{mg}$ ) & $\mathrm{R} \$ 0,49$ \\
Clonidina (ampola $150 \mathrm{\mu g}$ ) & $\mathrm{R} \$ 2,99$ \\
Cetoprofeno (ampola $100 \mathrm{mg})$ & $\mathrm{R} \$ 4,15$ \\
Óxido nitroso (m ${ }^{3}$ ) & $\mathrm{R} \$ 2,20$ \\
Oxigênio ( $\mathrm{m}^{3}$ ) & $\mathrm{R} \$ 2,05$ \\
Materiais descartáveis (conjunto) & $\mathrm{R} \$ 21,67$ \\
Sala de recuperação pós-anestésica (hora) & $\mathrm{R} \$ 3,66$ \\
\hline
\end{tabular}

Os dados foram expressos em freqüência, freqüência relativa (\%) ou médias \pm DP. As variáveis expressas na forma de freqüência foram analisadas estatisticamente pelos testes do Qui-quadrado ou Exato de Fisher. Dados expressos em médias foram analisados pelos testes de Kruskal-Wallis. Quando houve significância estatística $(p<0,05)$, seguiu-se comparação múltipla de Dunn's para análise entre os grupos.

\section{RESULTADOS}

Não houve diferença entre os grupos quanto aos dados demográficos avaliados (Tabela III). Os valores de pressão arterial e da freqüência cardíaca antes e após a administração da medicação pré-anestésica, antes e após a indução anestésica também não diferiram entre os grupos.

Tabela III - Dados Demográficos

\begin{tabular}{lccc}
\hline & Grupo S & Grupo C3 & Grupo C5 \\
\hline Idade * $^{*}$ & $28,13 \pm 10,39$ & $24,47 \pm 6,03$ & $30,87 \pm 12.51$ \\
Peso * & $63,20 \pm 10,90$ & $62,77 \pm 10,53$ & $60,25 \pm 14,93$ \\
Sexo & & & \\
$\quad$ Masculino & 9 & 8 & 7 \\
$\quad$ Feminino & 6 & 7 & 8 \\
\hline
\end{tabular}

*Valores expressos pela Média \pm DP

Revista Brasileira de Anestesiologia

Vol. 54, No 1, Janeiro - Fevereiro, 2004
Os tempos cirúrgico e anestésico foram semelhantes entre os grupos, bem como o tempo decorrido entre a interrupção do sevoflurano e a abertura ocular ao comando (fase I da recuperação anestésica). Já o tempo decorrido para alta ambulatorial (fase Il da recuperação), estimado entre a interrupção dos anestésicos e avaliação com nota 9 pelos critérios de recuperação adotados, foi maior no grupo C5 em relação ao grupo $S, p<0,01$ (Tabela IV). No final da segunda hora na SRPA, sete pacientes receberam alta no grupo $S$, enquanto nenhum paciente no grupo C5. Já no final de 3 horas de SRPA, todos os pacientes do grupo $S$ tinham recebido alta, enquanto 4 pacientes do grupo C5 ainda permaneciam em observação (Figura 1).

Tabela IV - Tempos Anestésicos, Cirúrgicos e Tempo Decorrido entre o Final da Administração de Halogenados e Abertura Ocular Espontânea e Extubação, bem como, Tempo entre o Final da Anestesia e Avaliação pelos Critérios de Aldrete-Kroulik com Desempenho 10 em Minutos (Média \pm DP)

\begin{tabular}{lccc}
\hline & Grupo S & Grupo C3 & Grupo C5 \\
\hline Tempo de cirurgia & $131,3 \pm 54,6$ & $119 \pm 64,9$ & $109,3 \pm 80,6$ \\
Tempo de anestesia & $153,3 \pm 43,1$ & $149,5 \pm 60,4$ & $140,8 \pm 73,5$ \\
Tempo para abertura ocular & $7,2 \pm 5,2$ & $7,3 \pm 1,8$ & $9,3 \pm 3,6$ \\
Tempo para extubação & $8,6 \pm 4,6$ & $8,2 \pm 2,8$ & $9,3 \pm 3,6$ \\
Tempo de SRPA & $131,4 \pm 60,8$ & $141,4 \pm 45,3$ & $190,3 \pm 68,3$ *
\end{tabular}

* Diferença estatística entre os grupos S e C5 pelo teste de Kruskal-Wallis com pós-teste de Dunn $(p<0,01)$

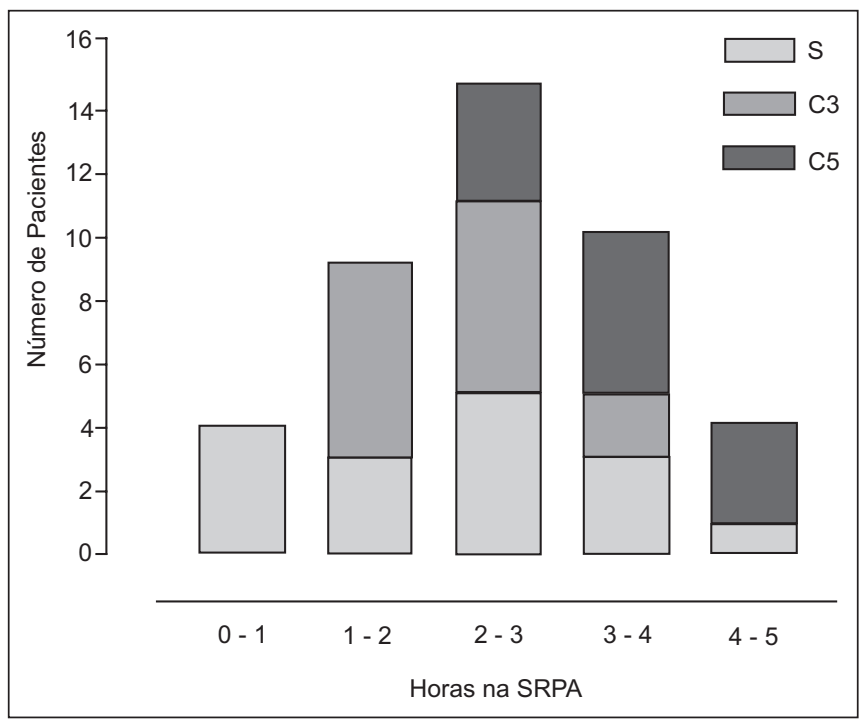

Figura 1 - Horas na Sala de Recuperação Pós-Anestésica

O consumo de sevoflurano em mililitro por minuto de cirurgia foram $0,54 \pm 0,14$ no grupo $S$ e 0,33 $\pm 0,09$ e 0,34 $\pm 0,13$ nos grupos C3 e C5, respectivamente. O consumo foi maior no grupo S que nos grupos C3 e C5, p < 0,001 e p <0,01, respectivamente. Não houve diferença entre os grupos C3 e C5 (Figura 1). 
Afreqüência geral de eventos adversos no peri-operatório foi semelhante nos três grupos, como demonstra a tabela V. Entretanto, a incidência de sonolência prolongada foi maior no grupo $\mathrm{C} 5$ em que quatro tiveram alta retardada, zero no grupo $S$ e um no grupo C3. A média e desvio-padrão do custo anestésicos em Reais foi 260,6 \pm 107,6, 171,4 \pm 55,23 e $170,8 \pm 39,76$ para os grupos S, C3 e C5, respectivamente. Apresentando diferença estatística entre os grupos S/C3 e S/C5 $(p<0,05)$.

Tabela V - Eventos Adversos no Peri-Operatório

\begin{tabular}{lccc}
\hline & Grupo S & Grupo C2 & Grupo C3 \\
\hline Hipotensão arterial & 0 & 0 & 1 \\
Hipotensão arterial postural & 0 & 2 & 3 \\
Disritmia & 3 & 1 & 0 \\
Bradicardia & 0 & 3 & 2 \\
Agitação & 3 & 0 & 0 \\
Sonolência prolongada & 0 & 1 & 4 \\
Vômito & 3 & 1 & 2 \\
Total & $9 / 15$ & $8 / 15$ & $12 / 15$ \\
\hline
\end{tabular}

\section{DISCUSSÃO}

A Anestesiologia é uma das especialidades que apresentou grande evolução técnico-científico nas últimas décadas. $O$ desenvolvimento de novas drogas anestésicas e correlatas, materiais específicos e técnicas geram aumento dos gastos diretos anestésicos, que podem ser atenuados com programas de contenção de custos ${ }^{9}$. O crescimento nos gastos resultou em maior conhecimento e preocupação por parte dos especialistas em diminuir os custos, mantendo a qualidade anestésica ${ }^{10}$. Estudo realizado com anestesiologistas canadenses demonstrou que $94 \%$ deles levam em consideração o custo quando praticam anestesia ${ }^{11}$. Em um país como o Brasil, carente de recursos para a área de saúde e dependente da importação de insumos para a indústria farmacêutica, a necessidade de programas para diminuição de custos é premente.

A análise de custo na área da saúde apresenta grandes dificuldades, muitas das quais relacionadas à multiplicidade de terminologias que envolvem este tipo de análise, bem como à escolha do método a ser empregado ${ }^{6}$. Neste estudo, a análise empregada foi do tipo custo-benefício, levando em consideração os custos diretos ( $p$. ex: preços de medicamentos), custos indiretos ou diretos associados (p. ex.: gasto com recuperação anestésica ${ }^{6}$ e benefício quantificado através do consumo de sevoflurano e do custo anestésico final. Porém, parte dos custos fixos não pode ser analisada, devido à falta de índices nacionais validados para o cálculo de cus- tos de depreciação de materiais (laringoscópio e outros), ventiladores e monitores. Esse tipo de custo excluído de nossa análise pode sofrer influência do tempo de utilização. Contudo, o tempo cirúrgico e anestésico foi semelhante entre os grupos e os recursos foram empregados de forma padronizada, minimizando o impacto em nossos resultados ${ }^{11}$. Nossos resultados mostram que a clonidina por via venosa diminuiu o consumo de sevoflurano entre $40 \%$ e $50 \%$. Estes resultados estão de acordo com trabalhos publicados na literatura, que mostram diminuição entre $40 \%$ e $60 \%$ no consumo de anestésicos ${ }^{5}$. Com o método utilizado, a diminuição no consumo de sevoflurano representou uma economia final de aproximadamente $35 \%$ nos gastos anestésicos. Este alto impacto que a economia de sevoflurano apresentou no custo anestésico final está em desacordo com dados obtidos em estudo que mostra que o custo de todos os agentes anestésicos utilizados contribui com no máximo $30 \%$ do custo anestésico final ${ }^{11}$. Contudo, este estudo foi realizado em país cujo gasto com recursos humanos apresenta alto impacto no custo final, diluindo a influência das medicações. Portanto, esta discordância pode ser explicada pelo fato de as medicações apresentarem preços internacionalizados; enquanto o gasto com salários e remuneração profissional são substancialmente menores em países como o Brasil. Outro importante fator que diferencia o estudo citado de nosso foi a não inclusão da remuneração paga ao anestesiologista, o que diminui o impacto da economia com medicação.

A dose de $5 \mu \mathrm{g} \cdot \mathrm{kg}^{-1}$ de clonidina não diminui o consumo além do observado para a dose de $3 \mu \mathrm{g} \cdot \mathrm{kg}^{-1}$. Por outro lado, além de não apresentar vantagem em relação à dose menor, prolonga de forma substancial a recuperação anestésica fase II, não se adequando ao regime ambulatorial.

A freqüência total de eventos adversos foi semelhante nos três grupos. Como era de se esperar, os pacientes que não receberam clonidina apresentaram mais agitação e com menos freqüência queixaram-se de boca seca ou tiveram hipotensão postural ${ }^{12}$.

Concluindo, a clonidina é uma droga coadjuvante anestésica que diminui o consumo de anestésicos. Sua utilização em anestesia geral com sevoflurano em regime ambulatorial pode representar uma economia de até $35 \%$ no custo anestésico final, excluindo a remuneração do anestesiologista. Nas condições aplicadas a este estudo, a dose de $3 \mu \mathrm{g} . \mathrm{kg}^{-1}$ apresentou-se como a melhor opção para o regime ambulatorial, pois não prolongou o despertar quando comparada ao grupo controle. A dose de $5 \mu \mathrm{g} . \mathrm{kg}^{-1}$ está relacionada à prolongamento da fase II de recuperação anestésica e maior incidência de hipotensão arterial postural. Devido às diferenças substanciais na composição de custos anestésicos entre países, torna-se evidente a necessidade de se realizar mais estudos brasileiros envolvendo diminuição de custos anestésicos. 


\section{Influence of Intravenous Clonidine in the Cost of Sevoflurane Anesthesia for Outpatient Middle Ear Procedures}

Renato Mestriner Stocche, TSA, M.D.; Luís Vicente Garcia, TSA, M.D.; Jyrson Guilherme Klamt, TSA, M.D.; Marlene Paulino dos Reis, M.D.; Daniela Rocha Gil, M.D.; Karin Luiza Magno Mesquita, M.D.

\section{INTRODUCTION}

Since the mid 1980s, there is a world trend to replace hospital by outpatient procedures. Early hospital discharge provides more comfort, autonomy and satisfaction for patients, in addition to decreasing the risk for nosocomial infections and the total cost of hospitalization ${ }^{1}$. Aiming at this early discharge, fast excretion anesthetic drugs are used in those patients. However, the high cost of such drugs may limit their use ${ }^{2}$. Clonidine is a central action $\alpha_{2}$-agonist which decreases sympathetic tone and exhibits sedative, antisialogogue, antiemetic and analgesic properties. It has also proven to be safe in the anesthetic practice, according to several studies. When used as preanesthetic medication, it decreases the need for intravenous and inhalational anesthetics ${ }^{3,4}$. Intravenous $1.5 \mu \mathrm{g} \mathrm{kg}^{-1}$ clonidine in outpatient procedures has not shown anxiolytic effects ${ }^{5}$. However, the effects of preanesthetic medication with clonidine on outpatient procedure anesthetic costs have not been evaluated, thus making necessary a cost-benefit ratio analysis ${ }^{6}$.

This study aimed at evaluating the cost-benefit ratio of preanesthetic medication with intravenous clonidine for general anesthesia with sevoflurane in outpatient procedures.

\section{METHODS}

After the Hospital das Clinicas, Ribeirão Preto Ethics Committee approval and patients their informed consent, 45 individuals aged 15 to 52 years, physical status ASA I and II, weighing 40 to $85 \mathrm{~kg}$, undergoing middle ear procedures were included in this double-blind, randomized and placebo controlled study. Exclusion criteria were patients with history of studied drugs allergy, with baseline heart rate lower than 50 bpm and those under chronic use of CNS depressing drugs, antidepressants, anticonvulsivants or drugs/alcohol abusers.

All patients were monitored with cardioscopy, pulse oximetry, capnometry and noninvasive blood pressure. After venous puncture with $20 \mathrm{G}$ catheter, hydration was standardized with $250 \mathrm{ml}$ rapid infusion followed by $6 \mathrm{ml} \cdot \mathrm{kg}^{-1} \cdot \mathrm{h}^{-1}$ lactated Ringer's infusion.

Patients were randomly distributed in 3 groups: Group C3 was given $3 \mu \mathrm{g} \cdot \mathrm{kg}^{-1}$ clonidine, Group C5 was given $5 \mu \mathrm{g} \cdot \mathrm{kg}^{-1}$ clonidine and Group $\mathrm{S}$ was given $0.9 \%$ sodium chloride as preanesthetic medication. Solutions containing $30 \mu \mathrm{g} \cdot \mathrm{ml}^{-1}$ clonidine for Group C3, $50 \mu \mathrm{g} \cdot \mathrm{ml}^{-1}$ clonidine for Group C5 and
$0.9 \%$ sodium chloride for Group S were prepared by an anesthesiologist not involved with the research. They were intravenously administered to all groups in a volume of $1 \mathrm{ml}$ solution for each $10 \mathrm{~kg}$ of body weight.

Inhalational anesthesia was induced 15 minutes after preanesthetic medication with sevoflurane at initial concentration of $3 \%$, followed by $0.5 \%$ increase at 15 -second intervals until loss of eyelid reflex. Alfentanil $\left(30 \mu \mathrm{g} \cdot \mathrm{kg}^{-1}\right)$ and pancuronium $\left(0.08 \mathrm{mg} . \mathrm{kg}^{-1}\right)$ were then administered. Anesthesia was maintained with sevoflurane, $\mathrm{N}_{2} \mathrm{O}$ and $\mathrm{O}_{2}(1: 1)$ aiming at maintaining MBP and $\mathrm{HR}$ within a $20 \%$ range above or below baseline values. All patients received $100 \mathrm{mg}$ ketoprofen in the beginning of the procedure.

At surgery completion, $100 \%$ oxygen was administered and the respiratory system was commuted to non-rebreathing. Tracheal extubation was performed when patients presented spontaneous and rhythmic ventilation for at least 30 seconds, maintaining $\mathrm{P}_{\mathrm{ET}} \mathrm{CO}_{2}$ below $50 \mathrm{mmHg}$, in addition to answering to verbal commands. Time elapsed between sevoflurane withdrawal (surgery completion) and eye opening at command (asked at 20-second intervals) was recorded, as was the time between sevoflurane withdrawal and tracheal extubation. Total sevoflurane consumption was also recorded.

The incidence of adverse events was recorded. Tachycardia was defined as heart rate (HR) above $100 \mathrm{bpm}$, bradycardia as $\mathrm{HR}$ below $50 \mathrm{bpm}$ and arterial hypotension as blood pressure below $30 \%$ of baseline value in two consecutive measurements. Adverse events were also recorded in the PACU (nausea, vomiting, mild or severe pain, urinary retention, postural arterial hypotension, sleepiness and dizziness). Oxygen nebulization ( $8 \mathrm{~L} \cdot \mathrm{min}^{-1}$ ) with Venturi mask was used until patients presented spontaneous emergence periods. Nausea and vomiting were treated with metochlopramide and the cost of this drug was added to total cost of anesthesia. Time for outpatient anesthetic discharge according to Chung's criteria ${ }^{8}$ was evaluated, namely, score 9 according to parameters scale described in table I.

Total anesthetic cost was calculated adding all expenditures with anesthetic drugs, oxygen, $\mathrm{N}_{2} \mathrm{O}$, disposable materials and cost of PACU stay (minutes). Monitors and ventilators depreciation and expenditures with reusable materials (drapes, pads, intermediaries and others) were not taken into consideration. The set of disposable materials contained one tracheal tube, one intravenous $20 \mathrm{G}$ catheter, one serum therapy equipment, $1 / 4$ tape roll, one plastic probe for aspiration and two pairs of latex gloves. Costs of drugs and disposable materials were obtained from Brasíndice published in July 20,2002 , discounting taxes built in the price. When drug or material had more than one manufacturer in Brasíndice, value considered for calculations was the arithmetic mean of values. Cost of PACU stay hour was obtained from the hospital's cost control center and corrected for July 2002. Oxygen and $\mathrm{N}_{2} \mathrm{O}$ cubic meter values were obtained directly from the supplier (price list) (Table II). 
Table I - Modified Criteria for Outpatient Anesthetic Discharge Vital Signs

$$
\begin{aligned}
& 2=\text { within } 20 \% \text { of baseline values } \\
& 1=\text { between } 20 \% \text { and } 40 \% \text { of baseline values } \\
& 0=>40 \% \text { of baseline values }
\end{aligned}
$$

Ambulation

$$
\begin{aligned}
& 2=\text { firm ambulation } / \text { w/o dizziness } \\
& 1=\text { assisted ambulation } \\
& 0=\text { no ambulation } / \text { dizziness }
\end{aligned}
$$

Nausea or Vomiting

$$
\begin{aligned}
& 2=\text { mild } \\
& 1=\text { moderate } \\
& 0=\text { severe }
\end{aligned}
$$

Pain

$$
\begin{aligned}
& 2=\text { mild } \\
& 1=\text { moderate } \\
& 0=\text { severe }
\end{aligned}
$$

Surgical Bleeding

$$
\begin{aligned}
& 2=\text { mild } \\
& 1=\text { moderate } \\
& 0=\text { severe }
\end{aligned}
$$

Table II - Costs of Materials and Anesthetic Drugs.

\begin{tabular}{lc}
\hline Drugs & Mean Price \\
\hline Alfentanil $(2.5 \mathrm{mg}$ vial $)$ & $\mathrm{R} \$ 15.29$ \\
Sevoflurane $(\mathrm{ml})$ & $\mathrm{R} \$ 2.62$ \\
Pancuronium $(4 \mathrm{mg}$ vial) & $\mathrm{R} \$ 4.88$ \\
Atropine $(0.25 \mathrm{mg}$ vial) & $\mathrm{R} \$ 0.45$ \\
Prostigmine $(0.5 \mathrm{mg}$ vial) & $\mathrm{R} \$ 0.49$ \\
Clonidine $(150 \mu \mathrm{g}$ vial $)$ & $\mathrm{R} \$ 2.99$ \\
Ketoprofen $(100 \mathrm{mg}$ vial) & $\mathrm{R} \$ 4.15$ \\
Nitrous oxide $\left(\mathrm{m}^{3}\right)$ & $\mathrm{R} \$ 2.20$ \\
Oxygen $\left(\mathrm{m}^{3}\right)$ & $\mathrm{R} \$ 2.05$ \\
Disposable material (set) & $\mathrm{R} \$ 21.67$ \\
Post-anesthetic recovery unit (hour) & $\mathrm{R} \$ 3.66$ \\
\hline
\end{tabular}

Data are shown in frequency, relative frequency (\%) or mean \pm SD. Variables expressed in frequency were statistically analyzed by Chi-square or Fisher's Exact tests. Data expressed in means were analyzed by Kruskal-Wallis test. In case of statistical significance $(p<0.05)$, Dunn's multiple comparisons test was used to between groups analysis.

\section{RESULTS}

There have been no differences between groups in demographics data (Table III). Blood pressure and heart rate before and after preanesthetic medication were also not differ- ent between them. Surgical and anesthetic times were similar between groups, as well as time elapsed from sevoflurane withdrawal to eye opening at command (phase I anesthetic recovery). However, time for outpatient discharge (phase II anesthetic recovery), calculated from anesthetic withdrawal to score 9 by adopted recovery criteria, was higher in group C5 as compared to Group S ( $p<0.01)$ (Table IV). At the end of 2 PACU hours, 7 Group S and no Group C5 patients were discharged. At the end of 3 PACU hours, all Group S patients had been discharged, while 4 Group C5 patients still remained in observation (Figure 1).

Table III - Demographics Data

\begin{tabular}{lccc}
\hline Variables & Group S & Group C3 & Group C5 \\
\hline Age * $^{*}$ & $28.13 \pm 10.39$ & $24.47 \pm 6.03$ & $30.87 \pm 12.51$ \\
Weight * & $63.20 \pm 10.90$ & $62.77 \pm 10.53$ & $60.25 \pm 14.93$ \\
Gender & & & \\
$\quad$ Male & 9 & 8 & 7 \\
Female & 6 & 7 & 8 \\
\hline
\end{tabular}

*Values expressed in Mean $\pm \mathrm{SD}$

Table IV - Anesthetic and Surgical Times, Time between Halogenate Withdrawal and Spontaneous Eye Opening and Extubation, as well as Time between End of Anesthesia and Evaluation by Aldrete-Kroulic Criteria with Performance 10 in Minutes (Mean \pm SD)

\begin{tabular}{lccc}
\hline & Group S & Group C3 & Group C5 \\
\hline Surgery time & $131.3 \pm 54.6$ & $119 \pm 64.9$ & $109.3 \pm 80.6$ \\
Anesthesia time & $153.3 \pm 43.1$ & $149.5 \pm 60.4$ & $140.8 \pm 73.5$ \\
Time for eye opening & $7.2 \pm 5.2$ & $7.3 \pm 1.8$ & $9.3 \pm 3.6$ \\
Time for extubation & $8.6 \pm 4.6$ & $8.2 \pm 2.8$ & $9.3 \pm 3.6$ \\
PACU time & $131.4 \pm 60.8$ & $141.4 \pm 45.3$ & $190.3 \pm 68.3$ * \\
\hline
\end{tabular}

* Statistical difference between Groups $\mathrm{S}$ and $\mathrm{C} 5$ by Kruskal-Wallis test with Dunn's post-test $(p<0.01)$

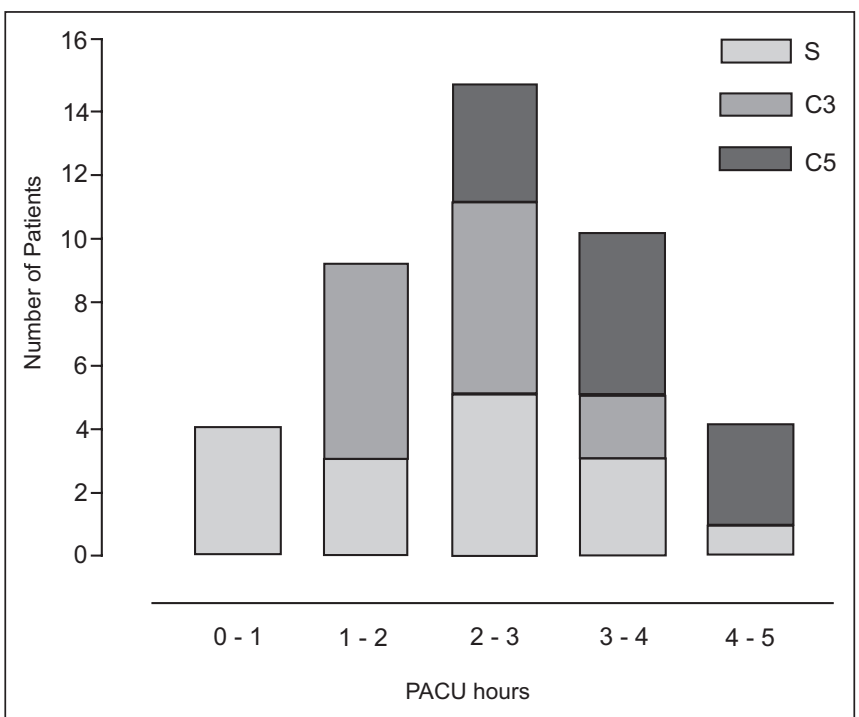

Figure 1 - Post-Anesthetic Recovery Unit Hours

Revista Brasileira de Anestesiologia Vol. 54, № 1, Janeiro - Fevereiro, 2004 
Sevoflurane consumption in milliliters per minute of surgery was $0.54 \pm 0.14,0.33 \pm 0.09$ and $0.34 \pm 0.13$ in Groups S, C3 and $\mathrm{C} 5$ respectively. Consumption was higher in Group $S$ as compared to Groups C3 and C5 ( $p<0.001$ and $p<0.01$ respectively). There was no difference between Groups $\mathrm{C} 3$ and C5 (Figure 1).

General perioperative adverse events incidence was similar for all groups, as shown in table V. However, the incidence of prolonged drowsiness was higher in Group C5 where 4 patients had delayed discharge, as compared to zero in Group S and one in Group C3. Mean anesthetic costs in Reals were $260.6 \pm 107.6,171.4 \pm 55.23$ and $170.8 \pm 39.76$ for Groups $S$, C3 and C5 respectively, with statistical difference between groups S/C3 and S/C5 ( $<<0.05)$.

Table V - Perioperative Adverse Events

\begin{tabular}{lccc}
\hline & Group S & Group C2 & Group C3 \\
\hline Arterial hypotension & 0 & 0 & 1 \\
Postural arterial hypotension & 0 & 2 & 3 \\
Arrhythmia & 3 & 1 & 0 \\
Bradycardia & 0 & 3 & 2 \\
Agitation & 3 & 0 & 0 \\
Prolonged sleepiness & 0 & 1 & 4 \\
Vomiting & 3 & 1 & 2 \\
Total & $9 / 15$ & $8 / 15$ & $12 / 15$ \\
\hline
\end{tabular}

\section{DISCUSSION}

Anesthesiology is a medical specialty which has shown a major technical-scientific evolution in the last decades. The development of new anesthetic drugs and alike, specific materials and techniques has increased direct anesthetic costs, which may be attenuated by cost contention programs ${ }^{9}$. Increased costs have resulted in better understanding and higher concern of specialists in decreasing them, while maintaining anesthetic quality ${ }^{10}$. A study performed with Canadian anesthesiologists has shown that $94 \%$ of them take into consideration costs when inducing anesthesia ${ }^{11}$. In a country such as Brazil, with few health care resources and depending on the import for the pharmaceutical industry, cost reduction programs are crucial.

Health care cost analysis poses major difficulties, many of them related to the diversity of terminologies involving this type of analysis, as well as to the method used ${ }^{6}$. This study adopted the cost-benefit ratio analysis, taking into consideration direct costs (e.g.: cost of drugs), indirect or associated direct costs (e.g.: cost of anesthetic recovery) ${ }^{6}$ and benefits quantified through sevoflurane consumption and total anesthetic costs. However, part of fixed costs could not be assessed due to the lack of validated Brazilian indices to calculate ventilators, monitors and materials depreciation costs (laryngoscope and others). These costs were excluded from our analysis and may be influenced by utilization time. How-

Revista Brasileira de Anestesiologia

Vol. 54, No 1, Janeiro - Fevereiro, 2004 ever, surgical and anesthetic times were similar between groups and resources were used in a standardized way to minimize the impact on our results ${ }^{11}$.

Our results have shown that intravenous clonidine decreased sevoflurane consumption in $40 \%$ to $50 \%$. These results are in line with the literature which shows $40 \%$ to $60 \%$ decrease in anesthetic consumption ${ }^{5}$. With our method, sevoflurane consumption decrease has represented final savings of approximately $35 \%$. This high impact of sevoflurane savings on total anesthetic costs does not agree with data obtained in a study which has shown that costs of all anesthetic agents contribute with no more than $30 \%$ of total anesthetic costs ${ }^{11}$. However, this study was carried out in a country where human resources expenditures have high impact on final costs, thus reducing drugs influence. So, this disagreement could be explained by the fact that drugs have international prices while expenditures with salaries and professional fees are substantially lower in countries such as Brazil. Another important factor differentiating the above-mentioned study from ours was the exclusion of anesthesiologists' fees, thus decreasing the impact of drug costs savings.

Clonidine at $5 \mu \mathrm{g} \cdot \mathrm{kg}^{-1}$ did not decrease consumption beyond what was observed with $3 \mu \mathrm{g} \cdot \mathrm{kg}^{-1}$. On the other hand, in addition to having no advantage as compared to the lower dose, it substantially prolonged phase II anesthetic recovery, being inadequate for outpatient procedures.

Total incidence of adverse events was similar for all groups. As expected, patients not receiving clonidine presented more restlessness and less dry mouth or postural arterial hypotension $^{12}$.

In conclusion, clonidine is an anesthetic adjuvant drug which decreases anesthetics consumption. Its use in outpatient general anesthesia with sevoflurane may represent savings of up to $35 \%$ in total anesthetic costs, excluding anesthesiologists' fees. In the conditions of our study, $3 \mu \mathrm{g} . \mathrm{kg}^{-1}$ clonidine was the best option for outpatient procedures because it has not delayed emergence as compared to control group. Clonidine at $5 \mu \mathrm{g} . \mathrm{kg}^{-1}$ was related to longer phase II anesthetic recovery and higher incidence of postural arterial hypotension. Due to substantial differences in anesthetic costs composition between countries, it is clear the need for further Brazilian studies involving anesthetic costs decrease.

\section{REFERÊNCIAS - REFERENCES}

01. Bain J, Kelly H, Snadden D et al - Day surgery in Scotland: patient satisfaction and outcomes. Qual Health Care, 1999;8:86-91.

02. Eger EI, White PF, Bogetz MS - Clinical and economic factors important to anaesthetic choice for day-case surgery. Pharmacoeconomics, 2000;17:245-262.

03. Howie MB, Hiestand DC, Jopling MW et al - Effect of oral clonidine premedication on anesthetic requirement, hormonal response, hemodynamics, and recovery in coronary artery bypass graft surgery patients. J Clin Anesth, 1996;8:263-272. 
04. Friedberg BL, Sigl JC - Clonidine premedication decreases propofol consumption during bispectral index (BIS) monitored propofol-ketamine technique for office-based surgery. Dermatol Surg, 2000;26:848-852.

05. Eberhart LH, Novatchkov N, Schricker T et al - Clonidine compared to midazolam for intravenous premedication for ambulatory procedures. A controlled double blind study in ASA I patients. Anaesthesiol Intensivmed Notfallmed Schmerzthe, 2000;35:388-393.

06. Watcha M, White PF - Economics of anesthetic practice. Anesthesiology, 1997;86:1170-1196.

07. Chung F - Practical issues in outpatient anaesthesia: discharge criteria - a new trend. Can J Anaesth, 1995;42:1056-1058.

08. Szocik JF, Learned DW - Impact of a cost containment program on the use of volatile anesthetics and neuromuscular blocking drugs. J Clin Anesth, 1994;6:378-382.

09. Hawkes C, Miller D, Martineau R et al - Evaluation of cost minimization strategies of anaesthetic drugs in a tertiary care hospital. Can J Anaesth, 1994;41:894-901.

10. Kantor G, Chung F - Anaesthesia drug cost, control and utilization in Canada. Can J Anesth, 1996;43:9-16.

11. Broadway PJ, Jones JG - A method of costing anaesthetic practice. Anaesthesia, 1995;50:56-63.

12. Stocche RM, Garcia LV, Klamt JG - Comparação entre nifedipina por via sub-lingual e clonidina por via venosa no controle de hipertensão peri-operatória em cirurgias de catarata. Rev Bras Anestesiol, 2002;52:426-433.

\section{RESUMEN}

Stocche RM, Garcia LV, Klamt JG, Reis MP, Gil DR, Mesquita KLM - Influencia de la Clonidina por Vía Venosa en el Costeo de Anestesia con Sevoflurano en Cirugías de Oído Medio en Régimen Ambulatorial

JUSTIFICATIVA Y OBJETIVOS: La clonidina es un agente $\alpha_{2}$ agonista que diminuye el consumo de anestésicos venosos e inhalatorios. Este estudio visó evaluar la relación costeo-beneficio de la medicación pre-anestésica con clonidina por vía venosa en anestesia general con sevoflurano en régimen ambulatorial.

MÉTODO: Se trata de estudio encubierto, aleatorio, controlado con placebo, realizado con pacientes con edad entre 15 y 52 años. Los pacientes fueron divididos en 3 grupos de 15: Grupo $S$ (placebo), C3 (clonidina $\left.3 \mu \mathrm{g} \cdot \mathrm{kg}^{-1}\right)$ y C5 $\left(5 \mu \mathrm{g} \cdot \mathrm{kg}^{-1}\right)$. La inducción anestésica fue hecha con sevoflurano, alfentanil (30 $\left.\mu \mathrm{g} \cdot \mathrm{kg}^{-1}\right)$ y pancuronio $\left(0,08 \mathrm{mg} \cdot \mathrm{kg}^{-1}\right)$. Fueron anotados la frecuencia de complicaciones, consumo de halogenados, tiempo de anestesia, tiempo de recuperación parte I y II. El análisis de gastos consideró gastos directos e indirectos.

RESULTADOS: No hubo diferencias entre los grupos en relación a los datos demográficos, frecuencia de complicaciones y tiempo para recuperación anestésica parte I. La recuperación anestésica parte II fue prolongada en el grupo $C 5(p<0,05)$. El consumo de sevoflurano por minuto de cirugía fue $0.54 \pm 0,14$ en el grupo $S$ y $0,33 \pm 0,09$ y $0,34 \pm 0,13$ en los grupos $C 3$ y $C 5$, respectivamente $(p<0,05)$. En los grupos que recibieron clonidina, el costeo fue diminuido en aproximadamente $35 \%$.

CONCLUSIONES: La clonidina $\left(3 \mu \mathrm{g} \cdot \mathrm{kg}^{-1}\right)$ por vía venosa diminuye el consumo de sevoflurano sin aumentar el tiempo de recuperación parte II. La dosis de $5 \mu \mathrm{g} \cdot \mathrm{kg}^{-1}$, a pesar de disminuir el consumo de sevoflurano, prolonga la recuperación parte II, no adecuándose al régimen ambulatorial. 